PROCEEDINGS OF THE

AMERICAN MATHEMATICAL SOCIETY

Volume 137, Number 1, January 2009, Pages 279-285

S 0002-9939(08)09473-2

Article electronically published on July 10, 2008

\title{
DEFORMATIONS OF HOLOMORPHIC LAGRANGIAN FIBRATIONS
}

\author{
JUSTIN SAWON \\ (Communicated by Ted Chinburg)
}

\begin{abstract}
Let $X \rightarrow \mathbb{P}^{n}$ be a $2 n$-dimensional projective holomorphic symplectic manifold admitting a Lagrangian fibration over $\mathbb{P}^{n}$. Matsushita proved that the fibration can be deformed in a codimension one family in the moduli space $\operatorname{Def}(X)$ of deformations of $X$. We extend his result by proving that if the Lagrangian fibration admits a section, then there is a codimension two family of deformations which also preserve the section.
\end{abstract}

\section{INTRODUCTION}

Let $X$ be a $2 n$-dimensional compact Kähler manifold. We say $X$ is a holomorphic symplectic manifold if it admits a closed two-form $\sigma$ of type $(2,0)$ which is non-degenerate in the sense that $\sigma^{\wedge n}$ trivializes the canonical bundle $K_{X}=\Omega^{2 n}$. Moreover, we call $X$ irreducible if $\mathrm{H}^{0}\left(X, \Omega^{2}\right)$ is one-dimensional and generated by $[\sigma]$. Huybrechts' notes in [7 provide a comprehensive introduction to the standard results on irreducible holomorphic symplectic manifolds.

Let us summarize what is known about fibrations on $X$. Suppose we have a proper (holomorphic) surjection $f: X \rightarrow B$ onto a complex space $B$ such that the general fibre is connected and $0<\operatorname{dim} B<2 n$. If $X$ is projective and $B$ is a normal variety, then Matsushita 9, 10, showed that

- every irreducible component of a fibre of $f$ is a (holomorphic) Lagrangian submanifold of $X$; in particular, it is $n$-dimensional,

- the generic fibre is an abelian variety,

- $B$ is $n$-dimensional and has only $\mathbb{Q}$-factorial log-terminal singularities,

- $K_{B}^{*}$ is ample and $B$ has Picard number one.

Moreover, if $B$ is smooth, then it has the same Hodge numbers as $\mathbb{P}^{n}$ (see [11). In particular, for $n=2$ the base is isomorphic to $\mathbb{P}^{2}$ (a similar result was obtain by Markushevich 8]). In general it is expected that $B$ should be isomorphic to $\mathbb{P}^{n}$. Huybrechts [7, Proposition 24.8] extended some of these results by dropping the projectivity assumption: he showed that if $X$ and $B$ are (smooth) Kähler manifolds, then

- every fibre of $f$ is (holomorphic) Lagrangian,

- every smooth fibre is a complex torus,

Received by the editors October 12, 2006, and, in revised form, March 2, 2007, and December 31, 2007.

2000 Mathematics Subject Classification. Primary 53C26, 14D06, 14J60.

(C)2008 American Mathematical Society

Reverts to public domain 28 years from publication 
- $B$ is $n$-dimensional and projective,

- $K_{B}^{*}$ is ample, and the Picard and second Betti numbers of $B$ both equal one.

In this paper we consider deformations of $f: X \rightarrow B$. It is known that the Kuranishi space $\operatorname{Def}(X)$ of deformations of $X$ is a smooth complex manifold of dimension $b_{2}-2$, where $b_{2}$ is the second Betti number of $X$. Under the assumptions that $X$ is projective and $B \cong \mathbb{P}^{n}$, Matsushita 11] proved that there is a codimension one submanifold of $\operatorname{Def}(X)$ parametrizing deformations of $X$ which are Lagrangian fibrations over $\mathbb{P}^{n}$. We include a proof of this result in Section 2 in order to set up our notation. Our main result is Theorem 4, we prove that if $f: X \rightarrow \mathbb{P}^{n}$ admits a section, then there is a codimension two submanifold of $\operatorname{Def}(X)$ parametrizing deformations which are Lagrangian fibrations and admit sections.

The Mordell-Weil group MW $(f)$ is the group of rational sections of $f: X \rightarrow B$. Recently Oguiso 14 found a formula for the rank of MW $(f)$, generalizing the Shioda-Tate formula for elliptic surfaces. Inspired by our techniques, he was able to produce examples with various Mordell-Weil ranks.

Let $X^{\prime}$ be an arbitrary irreducible holomorphic symplectic manifold. Can $X^{\prime}$ be deformed to a Lagrangian fibration? The answer in general is unknown. However, the question has been answered in the affirmative for all known examples of irreducible holomorphic symplectic manifolds; see Beauville 2] for the Hilbert schemes of points on a K3 surface, Debarre [5] for the generalized Kummer varieties, and Rapagnetta [15] for O'Grady's examples.

The author would like to thank Akira Fujiki, Daniel Huybrechts, and Daisuke Matsushita for useful conversations. Thanks are also given to Ryushi Goto for his invitation to Japan, where this result was obtained. The author was supported by NSF grant number 0305865 .

\section{Deforming FIBRATIONS}

Let $X$ be an irreducible holomorphic symplectic manifold of dimension $2 n$. For the following statements, see Section 22 of Huybrechts' notes in [7. Denote by

$$
\mathcal{X} \rightarrow(\operatorname{Def}(X), 0)
$$

the Kuranishi family parametrizing local deformations of $X=\mathcal{X}_{0}$. We think of $(\operatorname{Def}(X), 0)$ as the germ of a complex space. It is smooth (deformations are unobstructed) of dimension $b_{2}-2$, where $b_{2}$ is the second Betti number of $X$. Note that when we deform $X$ as a complex manifold it remains holomorphic symplectic and irreducible; for small deformations it also stays Kähler.

The germ $(\operatorname{Def}(X), 0)$ can be represented by a contractible open set, and therefore for each $t \in \operatorname{Def}(X)$ we can choose an isomorphism

$$
\psi_{t}: \mathrm{H}^{2}\left(\mathcal{X}_{t}, \mathbb{Z}\right) \rightarrow \mathrm{H}^{2}(X, \mathbb{Z})
$$

known as a marking. Let

$$
Q_{X}:=\left\{[\alpha] \in \mathbb{P}\left(\mathrm{H}^{2}(X, \mathbb{C})\right) \mid q_{X}(\alpha)=0 \text { and } q_{X}(\alpha+\bar{\alpha})>0\right\}
$$

be the period domain of $X$, where $q_{X}$ is the Beauville-Bogomolov quadratic form on $\mathrm{H}^{2}(X, \mathbb{Z}) \otimes \mathbb{C}$. Then by the Local Torelli Theorem (see Beauville [1]), the period map

$$
\mathcal{P}_{X}:(\operatorname{Def}(X), 0) \rightarrow\left(Q_{X},[\sigma]\right)
$$

which takes $t$ to $\left[\left(\psi_{t} \otimes \mathbb{C}\right)\left(\sigma_{t}\right)\right]$ is a local isomorphism. 
In this section we prove the following result.

Theorem 1 (Matsushita [11, Corollary 1.7). Let $X$ be a projective irreducible holomorphic symplectic manifold which admits a Lagrangian fibration $f: X \rightarrow$ $\mathbb{P}^{n}$ over projective space. There is a codimension one submanifold $\Delta^{f} \subset \operatorname{Def}(X)$ (containing zero) which parametrizes holomorphic symplectic manifolds $X^{\prime}$ which admit Lagrangian fibrations over $\mathbb{P}^{n}$.

Proof. Let $L:=f^{*} \mathcal{O}(1)$ be the pull-back of the hyperplane line bundle; then $f$ is the morphism

$$
\phi_{L}: X \rightarrow \mathbb{P}\left(\mathrm{H}^{0}(X, L)^{*}\right) \cong \mathbb{P}^{n}
$$

induced by the linear system of $L:=f^{*} \mathcal{O}(1)$. Matsushita [11] calculated the direct images of $\mathcal{O}_{X}$ under $f$ and found that $R^{j} f_{*} \mathcal{O}_{X} \cong \Omega_{\mathbb{P}^{n}}^{j}$. Together with the Leray spectral sequence this implies

$$
h^{0}(X, L):=\operatorname{dimH}^{0}(X, L)=n+1
$$

and all higher cohomology of $L$ vanishes.

Our aim is to extend $L$ and the map $\phi_{L}$ to deformations of $X$ for which the first Chern class $c_{1}:=c_{1}(L) \in \mathrm{H}^{2}(X, \mathbb{Z}) \cap \mathrm{H}^{1,1}(X)$ remains of type $(1,1)$. Let

$$
\Delta^{f}:=\left\{t \in \operatorname{Def}(X) \mid \psi_{t}^{-1}\left(c_{1}\right) \in \mathrm{H}^{2}\left(\mathcal{X}_{t}, \mathbb{Z}\right) \text { is of type }(1,1)\right\} .
$$

If $t \in \Delta^{f}$, then $\psi_{t}^{-1}\left(c_{1}\right)$ is orthogonal to $\sigma_{t}$ with respect to $q_{\mathcal{X}_{t}}$, or equivalently, $c_{1}$ is orthogonal to $\left(\psi_{t} \otimes \mathbb{C}\right)\left(\sigma_{t}\right)$ with respect to $q_{X}$. Therefore $\Delta^{f}$ maps isomorphically to a neighbourhood of $[\sigma]$ in

$$
Q_{X}^{f}:=\left\{[\alpha] \in Q_{X} \mid q_{X}\left(\alpha, c_{1}\right)=0\right\}
$$

which is of codimension one in $Q_{X}$. Hence $\Delta^{f}$ must be of codimension one in $\operatorname{Def}(X)$.

The exponential exact sequence on $\mathcal{X}_{t}$ gives

$$
\ldots \rightarrow 0 \rightarrow \mathrm{H}^{1}\left(\mathcal{X}_{t}, \mathcal{O}^{*}\right) \rightarrow \mathrm{H}^{2}\left(\mathcal{X}_{t}, \mathbb{Z}\right) \stackrel{\beta}{\rightarrow} \mathrm{H}^{2}\left(\mathcal{X}_{t}, \mathcal{O}\right) \rightarrow \ldots
$$

since $\mathrm{H}^{1}\left(\mathcal{X}_{t}, \mathcal{O}\right)$ vanishes. For $t \in \Delta^{f}, \psi_{t}^{-1}\left(c_{1}\right)$ is of type $(1,1)$ and so in the kernel of $\beta$. It therefore comes from a unique holomorphic line bundle $\mathrm{E}_{t} \in \mathrm{H}^{1}\left(\mathcal{X}_{t}, \mathcal{O}^{*}\right)$. Moreover, we can always choose a representative $(U, 0)$ of the germ $(\operatorname{Def}(X), 0)$ such that $U \cap \Delta^{f}$ is connected and simply-connected, so there exists a line bundle $\mathrm{E}$ over $\left.\mathcal{X}\right|_{\Delta^{f}}$, restricting to $\mathrm{E}_{t}$ on each fibre $\mathcal{X}_{t}$.

By the theorem on page 210 of Grauert and Remmert 6 , $h^{i}\left(\mathcal{X}_{t}, \mathrm{E}_{t}\right)$ is an upper semi-continuous function of $t \in \Delta^{f}$. So for $t$ in a neighbourhood of zero

$$
h^{i}\left(\mathcal{X}_{t}, \mathrm{七}_{t}\right) \leq h^{i}\left(\mathcal{X}_{0}, \mathrm{七}_{0}\right)=h^{i}(X, L) .
$$

For $i>0$ both sides must vanish, as we saw above that $h^{i}(X, L)=0$. On the other hand, the Euler characteristic

$$
\chi\left(\mathcal{X}_{t}, \mathrm{E}_{t}\right):=\sum_{i=0}^{2 n}(-1)^{i} h^{i}\left(\mathcal{X}_{t}, \mathrm{E}_{t}\right)
$$

is constant (it is given by the Hirzebruch-Riemann-Roch formula) and equal to $\chi(X, L)=n+1$. Therefore $h^{0}\left(\mathcal{X}_{t}, \mathrm{七}_{t}\right)=n+1$ for all $t$ in a neighbourhood of zero. The linear system of $\mathrm{E}_{t}$ therefore gives an a priori rational map

$$
\phi_{t}: \mathcal{X}_{t \rightarrow} \rightarrow \mathbb{P}\left(\mathrm{H}^{0}\left(\mathcal{X}_{t}, \mathrm{E}_{t}\right)^{*}\right) \cong \mathbb{P}^{n} \text {. }
$$


The specialization

$$
\phi_{L}=f: X \rightarrow \mathbb{P}\left(\mathrm{H}^{0}(X, L)^{*}\right) \cong \mathbb{P}^{n}
$$

is a morphism and thus has no base-points; therefore the linear system of $\mathrm{E}_{t}$ must also be base-point free for $t \in \Delta^{f}$ in a neighbourhood of zero. Moreover, $\phi_{t}: \mathcal{X}_{t} \rightarrow$ $\mathbb{P}^{n}$ is a Lagrangian fibration by the results of Matsushita (and Huybrechts) cited in the introduction.

\section{Fibrations with SeCtions}

We wish to look at deformations of a Lagrangian fibration over projective space which admits a section. We first prove that the existence of a section implies that $X$ is projective. The following argument is due to Campana and Oguiso [13].

Lemma 2. Let $f: X \rightarrow B$ be a Lagrangian fibration whose base $B$ is a normal projective variety. If $f: X \rightarrow B$ admits a rational multi-valued section (i.e., an $n$ dimensional subvariety $Y \subset X$ which maps surjectively to $B$ ), then $X$ is projective.

Proof. We know from Huybrechts [7, Proposition 24.8] that the generic fibre $X_{t}$ is a complex torus and holomorphic Lagrangian. Since $X$ is Kähler, $\sigma$ generates $\mathrm{H}^{0}\left(X, \Omega^{2}\right)$, and the restriction $\left.\sigma\right|_{X_{t}} \in \mathrm{H}^{0}\left(X_{t}, \Omega^{2}\right)$ vanishes, we can conclude that $X_{t}$ must be projective by a result of Voisin (which appears as Proposition 2.1 in Campana [4).

Let $a(B)$ and $a(Y)$ be the algebraic dimensions of $B$ and $Y$ respectively. Since $B$ is projective and $Y$ maps surjectively to $B$, we have

$$
n=\operatorname{dim} B=a(B) \leq a(Y) \leq \operatorname{dim} Y=n .
$$

Therefore we have equality, which implies that $Y$ is bimeromorphic to a projective variety; in particular, $Y$ is covered by complete algebraic curves. Since the generic fibre $X_{t}$ is also covered by complete algebraic curves, we conclude that any two generic points of $X$ are joined by a chain of complete algebraic curves. By a result of Campana [3], this implies that $X$ is Moishezon. Since $X$ is also Kähler, it must be projective [12].

Now suppose that the base $B$ is projective space and that $f: X \rightarrow \mathbb{P}^{n}$ admits a genuine section (i.e., a subvariety $Y \subset X$ which maps isomorphically to $\mathbb{P}^{n}$ ). Let $g: Y \hookrightarrow X$ be the inclusion; $Y$ must be holomorphic Lagrangian as

$$
\left.\sigma\right|_{Y} \in \mathrm{H}^{0}\left(Y, \Omega^{2}\right) \cong \mathrm{H}^{2,0}\left(\mathbb{P}^{n}\right)=0 .
$$

We will need the following result (note that in Voisin's paper $X$ is the Lagrangian submanifold of $Y$ ).

Theorem 3 (Voisin [19]). Let $X$ be a holomorphic symplectic manifold and let $g: Y \hookrightarrow X$ be a Lagrangian submanifold. Let $g_{t}^{*}$ be the composition

$$
g^{*} \circ\left(\psi_{t} \otimes \mathbb{C}\right): \mathrm{H}^{2}\left(\mathcal{X}_{t}, \mathbb{C}\right) \rightarrow \mathrm{H}^{2}(Y, \mathbb{C})
$$

where $\psi_{t}$ is a marking of $\mathcal{X}_{t}$. Then the inclusion $Y \hookrightarrow X$ deforms to a Lagrangian submanifold $\mathcal{Y}_{t} \hookrightarrow \mathcal{X}_{t}$ if and only if $g_{t}^{*}\left(\sigma_{t}\right)=0$.

We can rephrase this as follows. Let $L_{Y} \subset \mathrm{H}^{2}(X, \mathbb{C})$ be the orthogonal complement of kerg* with respect to the Beauville-Bogomolov quadratic form $q_{X}$. Since $\mathrm{H}^{2,0}(X) \subset \operatorname{ker} g^{*}$ and $L_{Y}$ can be defined over $\mathbb{Q}, L_{Y}$ must be of type $(1,1)$. Then 
$Y \hookrightarrow X$ deforms to $\mathcal{Y}_{t} \hookrightarrow \mathcal{X}_{t}$ if and only if $L_{Y}$ is preserved under the deformation, i.e., if and only if

$$
t \in \Delta:=\left\{t \in \operatorname{Def}(X) \mid \psi_{t}^{-1}\left(L_{Y}\right) \in \mathrm{H}^{2}\left(\mathcal{X}_{t}, \mathbb{C}\right) \text { is of type }(1,1)\right\} .
$$

We can now prove our main result.

Theorem 4. Let $X$ be an irreducible holomorphic symplectic manifold which admits a Lagrangian fibration $f: X \rightarrow \mathbb{P}^{n}$ over projective space and a global section. There is a codimension two submanifold $\Delta^{f s} \subset \operatorname{Def}(X)$ (containing zero) which parametrizes holomorphic symplectic manifolds $X^{\prime}$ which admit Lagrangian fibrations and global sections.

Proof. Lemma 2 implies $X$ is projective, and then Theorem 1 implies there exists a codimension one family $\Delta^{f} \subset \operatorname{Def}(X)$ over which the fibration deforms.

By Theorem 3 we also have

$$
\Delta^{s}:=\left\{t \in \operatorname{Def}(X) \mid \psi_{t}^{-1}\left(L_{Y}\right) \in \mathrm{H}^{2}\left(\mathcal{X}_{t}, \mathbb{C}\right) \text { is of type }(1,1)\right\}
$$

over which the Lagrangian submanifold $Y \hookrightarrow X$ deforms. Now $\Delta^{s}$ maps isomorphically to a neighbourhood of $[\sigma]$ in

$$
Q_{X}^{s}:=\left\{[\alpha] \in Q_{X} \mid q_{X}\left(\alpha, L_{Y}\right)=0\right\}=\left\{[\alpha] \in Q_{X} \mid \alpha \in \operatorname{ker} g^{*}\right\} .
$$

Since $Y \cong \mathbb{P}^{n}, \mathrm{H}^{2}(Y, \mathbb{C})$ and $L_{Y}$ are one-dimensional, so $Q_{X}^{s}$ and $\Delta^{s}$ are codimension one in $Q_{X}$ and $\operatorname{Def}(X)$ respectively.

Let $\Delta^{f s}:=\Delta^{f} \cap \Delta^{s}$. It maps isomorphically to a neighbourhood of $[\sigma]$ in

$$
Q_{X}^{f s}:=Q_{X}^{f} \cap Q_{X}^{s}=\left\{[\alpha] \in Q_{X} \mid q_{X}\left(\alpha, c_{1}\right)=0 \text { and } \alpha \in \operatorname{ker} g^{*}\right\} .
$$

Observe that $L:=f^{*} \mathcal{O}(1)$ must satisfy

$$
c_{1}(L)^{n+1}=0 \in \mathrm{H}^{2 n+2}(X, \mathbb{Z}),
$$

which implies $q_{X}\left(c_{1}\right)=0$ (see [7]) and thus $\left[c_{1}\right] \in Q_{X}^{f}$. On the other hand

$$
g^{*}\left(c_{1}\right)=c_{1}(\mathcal{O}(1)) \neq 0,
$$

so $c_{1} \notin \operatorname{ker} g^{*}$ and $\left[c_{1}\right] \notin Q_{X}^{s}$. This suffices to show that $Q_{X}^{f}$ and $Q_{X}^{s}$ intersect transversely, and thus $Q_{X}^{f s}$ and $\Delta^{f s}$ are codimension two in $Q_{X}$ and $\operatorname{Def}(X)$ respectively.

Finally, observe that if $t \in \Delta^{f s}$, then $\mathcal{X}_{t}$ is a Lagrangian fibration over $\mathbb{P}^{n}$ and it contains a deformation $\mathcal{Y}_{t}$ of $Y$. By specialization, $\mathcal{Y}_{t}$ is a section of the fibration. More precisely, the set of values $t$ for which $\mathcal{Y}_{t}$ maps isomorphically to $\mathbb{P}^{n}$ is open in $\Delta^{f s}$ and contains zero. This completes the proof.

Example. Let $S$ be a K3 surface which contains a smooth genus $g$ curve $C, g \geq 2$, such that $\operatorname{Pic} S$ is generated by $[C]$. Let $\mathcal{C} \rightarrow|C| \cong \mathbb{P}^{g}$ be the family of curves linearly equivalent to $C$. Then the compactified Jacobian

$$
J^{0}:=\overline{\operatorname{Jac}}^{0}\left(\mathcal{C} / \mathbb{P}^{g}\right)
$$

is a deformation of the Hilbert scheme of $g$ points on $S$ [2]. Moreover, $J^{0}$ is a Lagrangian fibration over $\mathbb{P}^{g}$ which admits a section. Observe that we can deform $S$ in a 19-dimensional family, while keeping the genus $g$ curve. These produce deformations of $J^{0}$ keeping the Lagrangian fibration and section. On the other hand, the space of deformations of the Hilbert scheme of points on $S$ is 21-dimensional. This is in agreement with Theorem 4

We find similar agreement for Lagrangian fibrations which are deformations of the generalized Kummer varieties (see Debarre [5] or the author's paper [16]). 
Remark. Let $f: X \rightarrow B$ be a Lagrangian fibration. If the fibres of $X$ are all reduced and irreducible, then there exists the (Altman-Kleiman) compactified relative Picard scheme

$$
P:=\overline{\operatorname{Pic}}^{0}(X / B) .
$$

If the fibres of $P$ are reduced and irreducible, we also have

$$
X^{0}:=\overline{\operatorname{Pic}}^{0}(P / B) .
$$

Both $P$ and $X^{0}$ admit global sections. Let $U$ and $U^{0}$ denote the open subsets of $X$ and $X^{0}$, respectively, given by removing all singular fibres; then $U$ is a torsor over $U^{0}$. In fact in cases where the singular fibres of $X$ are not too complicated, $X$ itself is a torsor over $X^{0}$ (by which we mean they are compactified group schemes over $B$ and locally isomorphic as fibrations); moreover $P$ and $X^{0}$ are holomorphic symplectic manifolds (see [18]). In [17] it was shown that torsors $X$ over $X^{0}$ are parametrized by the one-dimensional space $\mathrm{H}^{2}\left(P, \mathcal{O}^{*}\right)$ of gerbes on $P$, with $X^{0}$ being the unique fibration which admits a global section. This agrees with Theorem 1 and Theorem 4 which together imply that fibrations which admit sections must be codimension one inside the family of all fibrations.

\section{REFERENCES}

[1] A. Beauville, Variétés Kählériennes dont le 1ére classe de Chern est nulle, Jour. Diff. Geom. 18 (1983), 755-782. MR730926 (86c:32030)

[2] A. Beauville, Counting rational curves on K3 surfaces, Duke Math. J. 97 (1999), no. 1, 99-108. MR 1682284 (2000c:14073)

[3] F. Campana, Coréduction algébrique d'un espace analytique faiblement kählérien compact, Invent. Math. 63 (1981), no. 2, 187-223. MR610537 (84e:32028)

[4] F. Campana, Isotrivialité de certaines familles kählériennes de variétés non projectives, Math. Z. 252 (2006), no. 1, 147-156. MR2209156 (2007b:32022)

[5] O. Debarre, On the Euler characteristic of generalized Kummer varieties, Amer. J. Math. 121 (1999), no. 3, 577-586. MR.1738407 (2000m:14053)

[6] H. Grauert and R. Remmert, Coherent analytic sheaves, Grundlehren der Mathematischen Wissenschaften 265, Springer-Verlag, Berlin, 1984. MR755331 (86a:32001)

[7] M. Gross, D. Huybrechts, and D. Joyce, Calabi-Yau manifolds and related geometries, Springer Universitext, 2002. MR1963559 (2004c:14075)

[8] D. Markushevich, Lagrangian families of Jacobians of genus 2 curves, J. Math. Sci. 82 (1996), no. 1, 3268-3284. MR.1423641 (98b:14034)

[9] D. Matsushita, On fibre space structures of a projective irreducible symplectic manifold, Topology 38 (1999), no. 1, 79-83. Addendum, Topology 40 (2001), no. 2, 431-432. MR.1644091 (99f:14054) MR.1808227(2002d:32031)

[10] D. Matsushita, Equidimensionality of Lagrangian fibrations on holomorphic symplectic manifolds, Math. Res. Lett. 7 (2000), no. 4, 389-391. MR.1783616 (2001f:32041)

[11] D. Matsushita, Higher direct images of dualizing sheaves of Lagrangian fibrations, Amer. J. Math. 127 (2005), no. 2, 243-259. MR2130616 (2006b:14075)

[12] B. G. Moishezon, On n-dimensional compact complex manifolds having $n$ algebraically independent meromorphic functions. I, Izv. Akad. Nauk SSSR Ser. Mat. 30 (1966), 133-174. MR.0216522 (35:7355a)

[13] K. Oguiso, private communication.

[14] K. Oguiso, Shioda-Tate formula for an abelian fibered variety and applications, preprint math.AG/0703245.

[15] A. Rapagnetta, Topological invariants of O'Grady's six dimensional irreducible symplectic variety, Math. Zeitschrift 256 (2007), no. 1, 1-34. MR2282256 (2007j:14023)

[16] J. Sawon, Abelian fibred holomorphic symplectic manifolds, Turkish Jour. Math. 27 (2003), no. 1, 197-230. MR:1975339 (2004g:32021) 
[17] J. Sawon, Derived equivalence of holomorphic symplectic manifolds, in Algebraic Structures and Moduli Spaces, CRM Proc. Lecture Notes 38, AMS, 2004, 193-211. MR 2096146 (2005m:14075)

[18] J. Sawon, Twisted Fourier-Mukai transforms for holomorphic symplectic four-folds, Advances in Mathematics 218 (2008), no. 3, 828-864.

[19] C. Voisin, Sur la stabilité des sous-variétés lagrangiennes des variétés symplectiques holomorphes, Complex Projective Geometry, 294-303, LMS Lecture Note Ser. 179, Cambridge Univ. Press, 1992. MR:1201391 (94b:32029)

Department of Mathematics, Colorado State University, Fort Collins, Colorado 80523-1874

E-mail address: sawon@math.colostate.edu

$U R L$ : www . math.colostate.edu/ sawon 